Yakubu, Z., Loganathan, N., Hassan, A., Mardani, A., \& Streimikiene, D. (2019).

Financial and economic determinants of sustainable economic growth in Egypt, Nigeria and South Africa. Journal of International Studies, 12(4), 160-176. doi:10.14254/2071-

$8330.2019 / 12-4 / 11$

\title{
Financial and economic determinants of sustainable economic growth in Egypt, Nigeria and South Africa
}

\author{
Zakaria Yakubu \\ Azman Hashim International Business School, \\ Universiti Teknologi Malaysia, \\ Kuala Lumpur, Malaysia; \\ Department of Economics, \\ Kaduna State College of Education Gidan Waya, \\ Kafanchan, Kaduna State, Nigeria \\ zakyakdo@yahoo.com \\ Nanthakumar Loganathan \\ Azman Hashim International Business School, \\ Universiti Teknologi Malaysia, \\ Johor Bahru, Johor, Malaysia \\ n4nantha@yahoo.com
}

Asan Ali Golam Hassan

Azman Hashim International Business School, Universiti Teknologi Malaysia,

Kuala Lumpur, Malaysia

asanali@utm.my

\author{
*Abbas Mardani \\ Informetrics Research Group, Ton Duc Thang University, \\ Ho Chi Minh City, Vietnam \\ Faculty of Business Administration, Ton Duc Thang University, \\ Ho Chi Minh City, Vietnam. \\ *Corresponding Email:abbas.mardani@tdtu.edu.vn
}

\section{Dalia Streimikiene}

Lithuanian Institute of Agrarian Economics,

Vilnius, Lithuania

dalia.streimikiene@knf.vu.lt

Abstract. This study analyzes financial and economic determinants of sustainable economic performance using the quantile regression for the period from 1970 to 2016 for Egypt, Nigeria and South Africa. The main drivers of sustainable economic performance vary among the economies. It is driven by trade openness, government expenditure and political stability in Egypt. In South Africa, the 
reached. Also, there is complementarity between financial development and trade openness. This proves their advancement at the domestic financial markets in the direction to sustainable economic performance. Interestingly, Nigerian economy's sustainable growth is enhanced by capital account openness. Policies that will boast intra trade in Africa are encouraged as the AfCFTA has come to spur manufacturing activities on the continent. At the same time, emphasis should be set to improve financial and economic determinants of sustainable economic performance.

Keywords: bootstrap quantile regression, cointegration, economic growth.

\section{JEL Classification: F43, O47}

\section{INTRODUCTION}

A good financial system is the vehicle through which savings are mobilized to avail investment opportunities and this statement has made the seminal works of Shaw (1973) get greater acceptance in developing economies. These works have argued that lagging economies will have sustainable economic growth when they liberalize their financial systems. Many studies have also established the finance-growth relationship (Demirguc-Kunt \& Maksimovic, 1998; King \& Levine, 1993). Financial liberalization means reduction in the role of government and an increase in that of financial markets, while financial development is an increase in quantity and quality of financial services (Abiad et al., 2008). The indicators measuring financial liberalization are de jure changes in credit controls, interest rate control, removal of bank entry barriers, privatization, and removal of restrictions on international financial transactions. African countries, in a bid to promote economic growth when it was on decline during the 1980s, accepted the principles of the World Bank and IMF focused structurally adjusted economies (Fowowe, 2013). Financial liberalization is at the center of this reform policy. Financial liberalization can indeed improve financial development; however, their relationship is not that obvious. De Haan and Sturm (2017) asserted that a country with better scores on one dimension does not necessarily score well on the other.

Financial development influences on economic growth are palpable only at advanced level (Hermes \& Lesink, 2003; Danlami et al., 2017). At the initial stage of financial development, it might not have an impact on growth until it gets to an expected threshold. Ibrahim and Alagidede (2017) set such a threshold for financial development and they found that below the threshold the influence on economic growth was minimal, but after, it stimulates growth of sub-Sahara African countries. Thus, economies must improve their financial systems to attain sustainable economic growth. This matches the conclusion of Adeniyi et al. (2015) who stated that only after a certain threshold financial development impacts economic growth. On the contrary, Hook and Singh (2014) and also Cecchetti and Kharroubi (2012) indicated there is a nonlinear inverted U-shaped curve, on which at some point the impact of the financial development variable on growth becomes deleterious. Nevertheless, financial development remains to be prominent in stimulation of economic growth (Nawaz et al., 2019; Uddin et al., 2014; Polat et al., 2014; Korauš et al., 2017).

Financial liberalization magnifies the impact of corruption in an economy that has poor quality institutions which hinder capital account liberalization to stimulate economic growth. At the same time, in 
less corrupt economies, economic growth is enhanced via capital account liberalization (Kunieda et al., 2014). Thus, examination of the advancement in financial development to ascertain the presence of a threshold is imperative in this study. Prasad et al. (2003) examined the effect of extra financial openness in 76 developed and developing countries during the period of 1960-1999. They found no evidence to support the assertion that capital account openness assists in maintaining fluctuations in consumption growth in developing countries. Another study employed panel data on 45 developed and developing countries over the period of 1980-2002 (Bussiere \& Fratzscher, 2008), and these authors fail to identify any link between capital account openness and economic growth. However, they confirmed that capital account openness can only lead to overborrowing in the short term, though leading to a boom in the midterm, thus causing a recession. The place of capital account openness in developing countries cannot be neglected considering the contribution of Rajan \& Zingales (1998) with their hypothesis about simultaneous impacts of both capital and trade liberalization on financial development.

Onanuga (2016) also found that simultaneous openness of both capital account and trade is significant for the domestic financial market development in Nigeria., Pan et al. (2019) recently found that, the combined effects of financial development and trade liberalization has spurred the economic growth in most of the developing nations worldwide. Ahmed (2016) studies the impact of integrating financial market with financial development on growth for 30 Sub-Sahara African (SSA) countries, and the overall findings show an enhancing growth condition. According to Rajan and Zingales (2003), institutional quality is crucial toward the finance-growth relationship and countries which have good access to credit services tend to improve their economic performance Elkhuizen et al. (2017) contends that the variations on the impact of financial liberalization on economic growth between developed and developing economies is due to differences in terms of formal institutions. This was supported by Njikam (2017) and found a positive relationship between financial liberalization and economic growth in African countries.

To preview of empirical findings, Klein and Olivei, (2008) found that the capital account liberalization has a positive relationship with financial depth, in countries that have a good rule of law and sound formal institutions. This mainly because the capital account liberalization does not impact on economic growth directly, but via financial depth with good institutional factors. On the other hand, Assefa \& Mollick (2017) unearth that portfolio flows and foreign direct investment have a positive impact on economic growth depending on the domestic financial system. The factors that might lead to negative poor efficiency of a country are many among them are inappropriate government regulation and weak institutions.

Specifically, trade openness may lead to advances macroeconomic efficiency by giving access to new raw materials and products, low-cost, intermediate goods, a large market size and latest technologies (Herwartz \& Walle, 2014; Bilan et al., 2019a,b). According to Young (1991), trade openness might lessen the finance-growth relationship and aggregate economic performance if international trade disturbs the domestic industries. This is likely to be the case in some Africa countries with infant industries, as the African Continental Free Trade Agreement (AfCFTA) is aimed at increasing intra trade among the African countries, as only $15 \%$ of African exports go to other African countries now. In the long run period, AfCFTA is hoping for intra-trade within African countries and reasonable trade with Asian and European countries. Africa is going to be the manufacturing hub of the developing world and there is a need for all African countries to wake up to cease the chance of this robust policy mainly for emerging African countries such as Egypt, Nigeria and South Africa.

The remainder of this study is divided into the following: Section 2, data and empirical models, Section 3 data analysis and discussion, and Section 4 presents concluding remarks. 


\section{DATA AND EMPIRICAL MODELS}

This study focusses on the finance and economics determinants of economic sustainability for Egypt, Nigeria and South Africa. Based on the data availability from several sources, we select the yearly sample observations from 1970 to 2016. The variable descriptions, data source are shown in Table 1.

Table 1

Data sources and variable descriptions

\begin{tabular}{|l|l|l|}
\hline Variables & \multicolumn{1}{|c|}{ Descriptions } & \multicolumn{1}{|c|}{ Data source } \\
\hline RGDP & $\begin{array}{l}\text { Represents the real GDP per capita measures in US } \\
\text { currency at 2010 constant basic price. }\end{array}$ & World Development Indicators (2018) \\
\hline COP & $\begin{array}{l}\text { This represents capital account openness measures in } \\
\text { US currency, it is the sum of total foreign assets and } \\
\text { total foreign liabilities (\% of GDP). }\end{array}$ & $\begin{array}{l}\text { External Wealth of Nations Mark II Database } \\
\text { (see Lane, and Milesi-Ferretti, 2014) }\end{array}$ \\
\hline FD & $\begin{array}{l}\text { It represents financial development index proxy by } \\
\text { broad money, domestic credit to the private sector by } \\
\text { banks (as a \% of GDP) and the domestic credit } \\
\text { provided by the financial sector (as a \% of GDP). }\end{array}$ & World Development Indicators (2018) \\
\hline TOP & $\begin{array}{l}\text { It measures the countries volume of export and import } \\
\text { as measured in US currency (\% of GDP). }\end{array}$ & World Development Indicators (2018) \\
\hline GEX & $\begin{array}{l}\text { It stands for government expenditure on final goods } \\
\text { and services, excluding military expenditure (\% of } \\
\text { GDP). }\end{array}$ & World Development Indicators (2018) \\
\hline PST & $\begin{array}{l}\text { It represents the stability of political institutions. } \\
\text { Darshall et al. (2016) Polity IV Project }\end{array}$ \\
\hline
\end{tabular}

In order to investigate the relationship between financial, economic determinants and sustainable growth, we developed the following linear estimation model:

$$
R G D P_{t}=a_{0}+\beta_{1} C O P_{t}+\beta_{2} F D_{t}+\beta_{3} T O P_{t}+\beta_{4} G E X_{t}+\beta_{5} P S T_{t}+\beta_{6} F D_{t}^{2}+\varepsilon_{t}
$$

where, the $F D^{2}$ is the square of the series financial development as applied by Adeniyi et al. (2015), which is the indicator of financial development threshold and ${ }^{\varepsilon}$ is the stochastic error term. The GDP series is deflated to the constant basic price based on year 2000 (2010=100), COP is the capital account openness, FD stands for financial development index, TOP represents trade openness, GEX is the government expenditure and PST is the political stability variable. We followed Ahmed (2016) and Mubi (2012) approach by introducing the Principle Component Analysis (PCA) technique to create the financial development index (FD). We used three main financial indicators, such as broad money, domestic credit to the private sector by banks (as a \% of GDP) and the domestic credit provided by the financial sector (as a $\%$ of GDP) to develop the FD index. Furthermore, we also used the interaction effect between FD and COP; and the FD and TOP determine the effects on real GDP sustainability, as shown in Fig. 2.

$$
\begin{aligned}
R G D P_{t}= & a_{0}+\beta_{1} C O P_{t}+\beta_{2} F D_{t}+\beta_{3} T O P_{t}+\beta_{4} G E X_{t}+\beta_{5} P S T_{t}+\beta_{6}\left(F D_{t} \times C O P_{t}\right)+ \\
& \beta_{7}\left(F D_{t} \times T O P_{t}\right)+\mu
\end{aligned}
$$

Dealing with time series data required the test of a unit root in order to overcome the issue of spurious regression, as it is the case if the set of the observations is not stationary (Granger and Newbold, 1974). The presence of stationary indicates that the series of observations has a constant mean and variance (Ghosh, 2019; Simionescu et al., 2019). The unit root tests of Augmented Dickey Fuller (ADF) 
(Dickey and Fuller, 1981) and Phillips and Perron (PP) (Phillip and Perron, 1988) was employed in this study. Y. To detect the presence of structural break in the series the Zivot and Andrew (ZA) (Zivot and Andrew, 1992) test of an unknown break date was applied. There are three models for the test of unit root according to the ZA test. The Model 1 allows for one time change in the level of the series, Model 2, allows one-time change in the slope of the trend function; and Model 3, combines one time change in the level and the slope of the trend function of the series. The appropriate model to be used is the Model 3 because there is less loss of substantial power. Thus, the regression equation is as follows:

$$
\Delta y_{t}=c+\alpha y_{t-1}+\beta t+\theta D U_{t}+\gamma D T_{t}+\sum_{j=1}^{k} d_{j} \Delta y_{t-j}+\varepsilon_{t}
$$

where, $D U_{t}$ is the indicator of dummy variable for a mean shift at a possible break date that occur (TB), while $D T_{t}$, is the corresponding trend shift of the variable. The null hypothesis is $\alpha=0$ that the series $y_{t}$ has a unit root with a drift, which does not has any structural break, on the other hand, the alternative hypothesis $\alpha<0$ indicates that the series is a trend stationary process, that has one time break happening in a time unknown. The method accepts any point as a possible break date and estimate regression at any potential break date as they occur. The possible break date is taken among others break that one sided t-statistics is minimized for testing the ${ }^{\hat{\alpha}}=(\mathrm{a}-1)=1$.

In order to investigate the long run relationship between the estimated series, we used Bayer and Hanck (2013) combined cointegration test. This method tries to examine the cointegrating relation of the variables by making a combination of the Engle and Granger (1987), Johansen (1988), Boswijk (1994) and Banejee et al. (1998) cointegration methods. A joint t-statistics are created according to the four cointegration methodologies, as depicted in the following equations:

$$
\begin{aligned}
& E G-J O H=-2[\ln (P E G)+(P J O H)] \\
& E G-J O H-B O S-B D M=-2[ \\
& \ln (P E G)+(P J O H)+(P B O S)+(P B D M)]
\end{aligned}
$$

where, PEG, PJOH, PBOS and PBDM are the p-values of the test of cointegration at the individual level. If the estimated Fisher statistics larger than the critical values provided by Bayer and Hanck (2013), the null hypothesis of no cointegration will be rejected.

In long run cointegration analysis, we need to identify the long run coefficient value. Therefore, we apply the FMOLS and DOLS estimates in this study. FMOLS and DOLS methods, are proposed by Phillips and Hansen (1990); and Stock and Watson (1993) respectively. The FMOLS is a non-parametric method that corrects autocorrelation and heteroscedasticity by eliminating the correlation between the explanatory variables and the random term. While, DOLS is a parametric method that eliminate the correlation between the explained variables and the error terms by adding the lead and lag terms of the explanatory variables (Hu et al., 2018). In a nutshell, the FMOLS and DOLS are applied when the cointegration of the variables is $I(1)$. Thus, the long run coefficients will be confirmed by utilizing the FMOLS and DOLS.

We extent the long run cointegration models by incorporating the quantile regression. First, we used the quantile unit root test that gives emphasize on the various quantile effects introduced by Koenker and Xias's (2004). This test relies on the conditional quantile autoregression (AR) model and for RGDP quantile unit root test can be formulated as follows: 


$$
Q_{R G D P_{t}}\left(\tau \mid F_{t}\right)=\beta_{0}(\tau)+\beta_{1}(\tau) R G D P_{t-1}+\sum_{j=1}^{m} \beta_{j+1}(\tau) \Delta R G D P_{t-i}
$$

where, $Q_{R G D P_{t}}\left(\tau \mid F_{t}\right)$ stands for conditional distribution function, RGDP to a level $r \in(0,1)$, and $F_{t}$ means the accumulated information to the time $t$. The null hypothesis $H_{0}: \beta_{1}(\tau)=1$ for $(\tau)$ quantile. The Kolmgorov-Smirnov test suggested by Koenker and Xiao (2004) examine the nonstationary properties for a range of quantiles as indicate below:

$$
Q S K=\sup \sup \left|\tau_{n}(\tau)\right|
$$

where, $\tau_{n}(\tau)$ is the t-statistics, calculated at $\tau_{i} \in \Gamma$, thus, the QSK test is constructed using the maximum over $\tau$. The constraining distribution of the $\tau_{n}(\tau)$ and QKS test depend on nuisance parameters; thus, it is nonstandard. Furthermore, as $R G D P$ serves as the dependent variable and COP as the independent variable, the conditional quantile regression $\tau^{\text {th }}$ is expressed below:

$$
Q_{R G D P_{t}}(\tau \mid C O P)=\left\{b \mid F_{R G D P}(b \mid C O P) \geq \tau\right\}=\sum_{K}^{i} \beta_{K}(\tau) C O P_{K}=\chi^{1} \beta(\tau)
$$

where, $F_{R G D P}(b \mid C O P)$ represent the RGDP and COP conditional distribution function. While, the $\beta(\tau)$ are representing the regressed series at given quantiles $(\tau)$ relationship. Following Lin and Benjamin (2017 formulation, the estimated $\beta(\tau)$ for each quantile able to minimize the weighted deviation between the series, and this can be show as follows:

$$
\beta(\tau)=\operatorname{Argmin} \sum_{t=1}^{T}\left(\tau-1_{\left\{y t<\chi_{t}^{\prime} \beta(\tau)\right\}}\right)
$$

In this study, the quantile ${ }^{(\tau)}$ coefficient varies, and the equation $\beta_{K}^{(\tau)}$ are estimated to a range of $\tau=0.25,0.50,0.75$ and 0.90 .

\section{DATA ANALYSIS AND DISCUSSION}

Table 2 reports the summary statistics of the variables of this study For Egypt, the most volatile series is the FD (1.792), while the least is TOP $(0.257)$ in term of unconditional standard deviation values. The kurtosis coefficient of RGDP is the least with less than 2, but all other series are greater than 2. Also, all series except for COP and GEX are negatively skewed. In the case of the Nigerian economy, RGDP, FD and PST series are not normality distributed as the JB probability indicated. Similarly, the unconditional standard deviation of RGDP and FD is high, indicating they are most volatile with the coefficient of 2.202 and 1.817 respectively. The kurtosis value of FD is 5.045 followed by that of the TOP 2.198 and the kurtosis of all series are not 0 , it implies the absence of normal distribution in of the series. 
Looking at South Africa, the RGDP, COP, GEX and PST series are not normally distributed based on the JB test. The standard deviation of FD and PST are the highest 1.826 and 1.328 respectively; and the skewness of RGDP, COP and FD is exhibiting positive tails with kurtosis less than 2 . On the other hand, other series exhibit negative tails with kurtosis greater than 2 , implying the series is abnormally distributed.

Table 2

Summary of statistics

\begin{tabular}{|c|c|c|c|c|c|c|c|}
\hline & Mean & Median & Maxim & Min & Std. Dev. & Skew. & $\begin{array}{l}\text { JB test } \\
\text { (p-value) }\end{array}$ \\
\hline \multicolumn{8}{|c|}{ County: Egypt } \\
\hline RGDP & 8.637 & 8.286 & 9.629 & 7.808 & 0.653 & 0.340 & 0.051 \\
\hline $\mathrm{COP}$ & -0.153 & -0.053 & 0.555 & -1.234 & 0.464 & -0.768 & 0.098 \\
\hline FD & 0.000 & 0.242 & 2.960 & -3.517 & 1.792 & -0.386 & 0.403 \\
\hline TOP & 3.900 & 3.925 & 4.409 & 3.402 & 0.257 & -0.103 & 0.447 \\
\hline GEX & 2.656 & 2.533 & 3.340 & 2.331 & 0.306 & 0.864 & 0.038 \\
\hline PST & 2.886 & 3.401 & 3.970 & 0.000 & 1.210 & -1.241 & 0.002 \\
\hline \multicolumn{8}{|c|}{ Country: Nigeria } \\
\hline RGDP & 9.686 & 8.368 & 12.865 & 7.284 & 2.202 & 0.372 & 0.046 \\
\hline COP & -0.451 & -0.592 & 0.586 & -1.209 & 0.532 & 0.407 & 0.173 \\
\hline FD & -0.000 & -0.201 & 6.250 & -2.975 & 1.817 & 1.079 & 0.000 \\
\hline TOP & 3.782 & 3.859 & 4.404 & 2.977 & 0.394 & -0.516 & 0.188 \\
\hline GEX & 2.291 & 2.320 & 2.887 & 1.576 & 0.405 & -0.234 & 0.104 \\
\hline PST & 1.663 & 1.946 & 2.772 & 0.000 & 0.859 & -0.749 & 0.078 \\
\hline \multicolumn{8}{|c|}{ Country: South Africa } \\
\hline RGDP & 9.676 & 9.405 & 11.133 & 8.228 & 0.969 & 0.237 & 0.091 \\
\hline COP & -0.080 & -0.325 & 0.853 & -0.703 & 0.509 & 0.531 & 0.069 \\
\hline FD & -0.000 & -0.472 & 4.134 & -2.393 & 1.826 & 0.496 & 0.105 \\
\hline TOP & 3.948 & 3.944 & 4.289 & 3.624 & 0.150 & -0.190 & 0.751 \\
\hline GEX & 2.830 & 2.912 & 3.035 & 2.408 & 0.185 & -0.911 & 0.028 \\
\hline PST & 3.094 & 3.091 & 4.407 & 0.000 & 1.328 & -0.843 & 0.058 \\
\hline
\end{tabular}

Table 3 shows the results of the stationary properties of the variables and this is the preliminary requirement of the quantile regression estimation. In terms of the traditional tests for unit root, we found both of the ADF and PP tests agree that all variables involve in this study are integrated of order $I(1)$. Since none of the variables are integrated of order $I(0)$, the long run cointegration and quantile regression analysis can be employed in this study. Since there was consistency within the traditional unit root tests for all variables, we used to test the unknown break of unit root using the ZA test. In our study, the unknown break date revealed there is a structural break in real GDP per capita in 1987 for Egypt, while for Nigeria and South Africa it is in 2004 and 1996 respectively. The break date of Egypt in 1987 was mainly due to the collapse in windfall revenue following the 1985 until1986 oil price crash that led to the unsustainability of prevailing fiscal policy. We realize that, the fiscal deficit escalated to an average rate of $15 \%$ of GDP, an automatic shock absorber was set through expansionary monetary policy, which resulted in inflation rates above $20 \%$. On the part of Nigeria, in 2004, there was sudden declined on oil price after the oil boom as mentioned through Onyeiwu and Oladimeji (2018) previous empirical findings. In the case of South Africa, with the turn of democracy in 1994, the country's economy was overwhelmed with sanctions busting, that promote uncompetitive exports at huge cost to the state. Similarly, transformation 
in the industry via Broad-Based Black Economic Empowerment (BBBEE), in order to create an inclusive economy by supporting small businesses and co-operation in the early 2000s (Fotoyi, et al., 2016).

Table 3

The unit root test results

\begin{tabular}{|c|c|c|c|c|c|c|}
\hline & \multicolumn{3}{|c|}{ At level } & \multicolumn{3}{|c|}{ At first difference } \\
\hline & ADF & PP & $\mathbf{Z A}$ & ADF & PP & ZA \\
\hline \multicolumn{7}{|c|}{ Country: Egypt } \\
\hline RGDP & -2.040 & -1.713 & $\begin{array}{c}-4.309 \\
T_{B}:_{1987)}\end{array}$ & $-4.174^{*}$ & $-4.178^{* *}$ & $\begin{array}{l}4.925^{* * *} \\
T_{B}:_{1993)}\end{array}$ \\
\hline COP & -2.251 & -1.869 & $\begin{array}{c}-3.211 \\
T_{B}:_{1979)}\end{array}$ & $-5.341 *$ & $-5.258^{*}$ & $\begin{array}{c}-5.660^{*} \\
T_{B}:_{1990)}\end{array}$ \\
\hline FD & -1.886 & $\begin{array}{c}-1.978 \\
\end{array}$ & $\begin{array}{c}-3.453 \\
T_{B}:_{1981)}\end{array}$ & $-3.689 * *$ & $-3.978^{* *}$ & $\begin{array}{l}-4.972^{* *} \\
T_{B}:_{2007)}\end{array}$ \\
\hline TOP & -2.125 & -2.125 & $\begin{array}{c}-2.860 \\
T_{B}:_{2009)}\end{array}$ & $-5.868^{*}$ & $-5.868^{*}$ & $\begin{array}{c}-6.352^{*} \\
T_{B}:_{1988)}\end{array}$ \\
\hline GEX & -1.620 & -1.450 & $\begin{array}{c}-2.536 \\
T_{B}:_{1987)}\end{array}$ & $-8.771^{*}$ & $-8.944^{*}$ & $\begin{array}{c}-6.353^{*} \\
T_{B}:_{1988)}\end{array}$ \\
\hline PST & -2.512 & -1.676 & $\begin{array}{c}-3.993 \\
T_{B}:_{1998)}\end{array}$ & $-7.248^{*}$ & $-7.852^{*}$ & $\begin{array}{c}-8.398^{*} \\
T_{B}:_{2005)}\end{array}$ \\
\hline \multicolumn{7}{|c|}{ Country: Nigeria } \\
\hline RGDP & -1.319 & -1.075 & $\begin{array}{c}-3.222 \\
T_{B}:{ }_{2004)}\end{array}$ & $-4.210^{*}$ & $-4.302^{*}$ & $\begin{array}{c}5.877^{*} \\
T_{B}:_{1981)}\end{array}$ \\
\hline COP & -1.978 & -1.978 & $\begin{array}{c}-4.013 \\
T_{B}:_{1983)}\end{array}$ & $-5.852^{*}$ & $-5.831^{*}$ & $\begin{array}{c}-6.147^{*} \\
T_{B}:_{1990)}\end{array}$ \\
\hline FD & -2.869 & -2.561 & $\begin{array}{c}-3.831 \\
T_{B}:_{1980)}\end{array}$ & $-6.143^{*}$ & $-11.736^{*}$ & $\begin{array}{c}-6.242^{*} \\
T_{B}:{ }_{1997)}\end{array}$ \\
\hline TOP & -1.594 & -1.517 & $\begin{array}{c}-2.172 \\
T_{B}:_{1989)}\end{array}$ & $-8.824^{*}$ & $-8.761^{*}$ & $\begin{array}{l}-10.423^{*} \\
T_{B}:{ }_{1987}\end{array}$ \\
\hline GEX & -2.811 & -2.963 & $\begin{array}{c}-3.767 \\
T_{B}:{ }_{1994)}\end{array}$ & $-7.430^{*}$ & $-7.456^{*}$ & $\begin{array}{l}-8.107^{*} \\
T_{B}:{ }_{1992)}\end{array}$ \\
\hline PST & -2.510 & -2.712 & $\begin{array}{c}-3.745 \\
T_{B}:_{1978)}\end{array}$ & $-6.342^{*}$ & $-6.384^{*}$ & $\begin{array}{c}-7.260^{*} \\
T_{B}:_{1980)}\end{array}$ \\
\hline RGDP & -2.167 & -1.799 & $\begin{array}{c}\text { Oountry: Sout } \\
-3.423 \\
T_{B}:\end{array}$ & $-4.686^{*}$ & $-4.544^{*}$ & $\begin{array}{c}-6.342^{*} \\
T_{B}{ }^{2}\end{array}$ \\
\hline $\mathrm{COP}$ & -1.875 & -1.891 & $T_{B}^{-3.610}$ & $-6.755^{*}$ & $-6.755^{*}$ & $T_{B}^{-6.011^{*}}$ \\
\hline FD & -1.263 & -1.774 & $T_{B}^{-6.097}$ & $-10.734^{*}$ & $-17.277^{*}$ & $\begin{array}{c}-7.504^{*} \\
T_{B}:_{1992)}\end{array}$ \\
\hline TOP & -1.982 & -1.955 & $\begin{array}{c}-3.405 \\
T_{B}:{ }_{1982)}\end{array}$ & $-6.063^{*}$ & $-6.357^{*}$ & $\begin{array}{l}{ }^{-6.731^{*}} \\
T_{B}:_{1993)}\end{array}$ \\
\hline GEX & -1.867 & -1.695 & $\begin{array}{c}-4.315 \\
T_{B}:{ }_{1982)}\end{array}$ & $-6.823^{*}$ & $-7.077^{*}$ & $T_{B}^{-6.995^{*}}$ \\
\hline PST & -1.810 & -1.810 & $\begin{array}{c}-2.797 \\
T_{B}:{ }_{1995)}\end{array}$ & $-6.376^{*}$ & $-6.376^{*}$ & $\begin{array}{c}-7.304^{*} \\
T_{B}:{ }_{1994)}\end{array}$ \\
\hline
\end{tabular}

Note: *, **, *** means at 1, 5 and $10 \%$, respectively $\mathrm{T}_{\mathrm{B}}$ represents the break year of the Zivot and Andrew (1992) unit root test. 
The quantile unit root test estimation results reported in Table 4. The behavior of each variable in specific quantile is examined by the value of the intercept $\alpha\left(\tau_{0}\right)$ and the autoregressive coefficient $\alpha\left(\tau_{1}\right)$. The estimated results of $\alpha\left(\tau_{0}\right)$ and $\alpha\left(\tau_{1}\right)$ represent the constant term and autoregressive coefficient. While the QKS test indicates the mean reverting in the estimated series. The intercept $\alpha\left(\tau_{0}\right)$ investigate the magnitude of the shock observed within the ${ }^{\tau}$ quantile that affects the series.

Table 4

Quantile unit root results

\begin{tabular}{|c|c|c|c|c|c|c|c|c|c|c|c|c|}
\hline & \multicolumn{4}{|c|}{ Egypt } & \multicolumn{4}{|c|}{ Nigeria } & \multicolumn{4}{|c|}{ South Africa } \\
\hline & q25 & q50 & q75 & $\mathrm{q} 90$ & q25 & q50 & q75 & $\mathrm{q} 90$ & q25 & q50 & q75 & $\mathrm{q} 90$ \\
\hline \multicolumn{13}{|c|}{ Panel 1: RGDP } \\
\hline$\alpha\left(\tau_{0}\right)$ & \begin{tabular}{|l|}
-0.068 \\
$(0.113)$
\end{tabular} & $\begin{array}{l}-0.011 \\
(0.363)\end{array}$ & $\begin{array}{c}0.013 \\
(0.346)\end{array}$ & $\begin{array}{c}0.049 \\
(0.205)\end{array}$ & $\begin{array}{c}-0.296^{*} \\
(0.000)\end{array}$ & $\begin{array}{l}-0.068 \\
(0.113)\end{array}$ & $\begin{array}{c}0.059 \\
(0.278)\end{array}$ & $\begin{array}{c}0.123 \\
(0.430)\end{array}$ & $\begin{array}{c}- \\
0.129 * * * \\
(0.082)\end{array}$ & $\begin{array}{l}-0.033 \\
(0.328)\end{array}$ & $\begin{array}{l}-0.002 \\
(0.489)\end{array}$ & \begin{tabular}{|c|}
0.117 \\
$(0.248)$
\end{tabular} \\
\hline$\alpha\left(\tau_{1}\right)$ & \begin{tabular}{|c|}
0.975 \\
$(0.359)$ \\
\end{tabular} & $\begin{array}{c}0.967 \\
(0.197)\end{array}$ & $\begin{array}{c}0.976 \\
(0.248) \\
\end{array}$ & $\begin{array}{c}0.957 \\
(0.229) \\
\end{array}$ & $\begin{array}{c}0.963 \\
(0.138)\end{array}$ & $\begin{array}{c}0.972 \\
(0.140)\end{array}$ & $\begin{array}{c}1.015 \\
(0.356) \\
\end{array}$ & $\begin{array}{c}1.013 \\
(0.456)\end{array}$ & $\begin{array}{c}0.996 \\
(0.476)\end{array}$ & $\begin{array}{c}1.012 \\
(0.396) \\
\end{array}$ & $\begin{array}{c}1.014 \\
(0.407) \\
\end{array}$ & \begin{tabular}{|c|}
0.993 \\
$(0.473)$ \\
\end{tabular} \\
\hline$\alpha\left(\tau_{n}\right)$ & \begin{tabular}{|l|}
-0.898 \\
$(0.520)$ \\
\end{tabular} & $\begin{array}{l}-1.502 \\
(0.300) \\
\end{array}$ & $\begin{array}{l}-1.080 \\
(0.420) \\
\end{array}$ & $\begin{array}{l}-1.232 \\
(0.290) \\
\end{array}$ & $\begin{array}{l}-1.266 \\
(0.170) \\
\end{array}$ & $\begin{array}{l}-1.320 \\
(0.190) \\
\end{array}$ & $\begin{array}{c}0.759 \\
(0.930) \\
\end{array}$ & $\begin{array}{c}0.040 \\
(0.810) \\
\end{array}$ & $\begin{array}{l}-0.119 \\
(0.790) \\
\end{array}$ & $\begin{array}{c}0.596 \\
(0.940) \\
\end{array}$ & $\begin{array}{c}0.376 \\
(0.960) \\
\end{array}$ & \begin{tabular}{|l|}
-0.115 \\
$(0.860)$ \\
\end{tabular} \\
\hline $\begin{array}{l}\text { QSK- } \\
\text { stat }\end{array}$ & \multicolumn{4}{|c|}{$1.502[5.129]$} & \multicolumn{4}{|c|}{$2.366[4.987]$} & \multicolumn{4}{|c|}{0.951 [5.992] } \\
\hline \multicolumn{13}{|c|}{ Panel 2: COP } \\
\hline$\alpha\left(\tau_{0}\right)$ & $\begin{array}{c}- \\
0.093 * * \\
(0.022)\end{array}$ & $\begin{array}{c}0.006 \\
(0.396)\end{array}$ & $\begin{array}{c}0.058^{* *} \\
(0.036)\end{array}$ & $\begin{array}{c}0.158^{* *} \\
(0.021)\end{array}$ & $\begin{array}{l}-0.204^{*} \\
(0.000)\end{array}$ & $\begin{array}{l}-0.020 \\
(0.311)\end{array}$ & $\begin{array}{c}0.092^{* *} \\
(0.047)\end{array}$ & $\begin{array}{l}0.303^{*} \\
(0.004)\end{array}$ & $\begin{array}{c}- \\
0.078^{* * *} \\
(0.055) \\
\end{array}$ & $\begin{array}{c}0.006 \\
(0.471)\end{array}$ & $\begin{array}{c}0.085 \\
(0.191)\end{array}$ & $\begin{array}{l}0.188^{* *} \\
(0.047)\end{array}$ \\
\hline$\alpha\left(\tau_{1}\right)$ & $\begin{array}{c}0.979 \\
(0.415)\end{array}$ & $\begin{array}{l}0.837^{*} \\
(0.001)\end{array}$ & $\begin{array}{l}0.767^{*} \\
(0.000)\end{array}$ & $\begin{array}{c}0.722^{* *} \\
(0.038)\end{array}$ & $\begin{array}{c}0.871 \\
(0.158)\end{array}$ & $\begin{array}{c}0.873^{* * *} \\
(0.058)\end{array}$ & $\begin{array}{c}0.831 * * * \\
(0.060)\end{array}$ & $\begin{array}{c}0.815 \\
(0.217)\end{array}$ & $\begin{array}{c}0.973 \\
(0.358)\end{array}$ & $\begin{array}{c}0.996 \\
(0.486)\end{array}$ & $\begin{array}{c}0.993 \\
(0.470)\end{array}$ & $\begin{array}{c}0.987 \\
(0.468) \\
\end{array}$ \\
\hline$\alpha\left(\tau_{n}\right)$ & $\begin{array}{l}-0.217 \\
(0.680) \\
\end{array}$ & $\begin{array}{c}-2.701^{* *} \\
(0.030)\end{array}$ & $\begin{array}{l}-4.218^{*} \\
(0.000)\end{array}$ & $\begin{array}{l}-1.341 \\
(0.320)\end{array}$ & $\begin{array}{l}-0.839 \\
(0.500)\end{array}$ & $\begin{array}{l}-1.362 \\
(0.290)\end{array}$ & $\begin{array}{l}-1.164 \\
(0.180)\end{array}$ & $\begin{array}{l}-0.539 \\
(0.670)\end{array}$ & $\begin{array}{l}-0.542 \\
(0.540)\end{array}$ & $\begin{array}{l}-0.067 \\
(0.810)\end{array}$ & $\begin{array}{l}-0.120 \\
(0.830)\end{array}$ & $\begin{array}{l}-0.071 \\
(0.730)\end{array}$ \\
\hline $\begin{array}{l}\text { QSK- } \\
\text { stat }\end{array}$ & \multicolumn{4}{|c|}{4.218 [4.383] } & \multicolumn{4}{|c|}{1.863 [4.484] } & \multicolumn{4}{|c|}{0.618 [3.825] } \\
\hline \multicolumn{13}{|c|}{ Panel 3: FD } \\
\hline$\alpha\left(\tau_{0}\right)$ & $\begin{array}{c}- \\
0.286^{* *} \\
(0.029)\end{array}$ & $\begin{array}{c}0.073 \\
(0.322)\end{array}$ & $\begin{array}{l}0.250^{*} \\
(0.014)\end{array}$ & $\begin{array}{c}0.403^{* *} \\
(0.023)\end{array}$ & $\begin{array}{c}-0.753^{*} \\
(0.005)\end{array}$ & $\begin{array}{c}0.127 \\
(0.210)\end{array}$ & $\begin{array}{l}0.619^{*} \\
(0.002)\end{array}$ & $\begin{array}{l}1.308^{*} \\
(0.002)\end{array}$ & $\begin{array}{l}-0.245 \\
(0.134)\end{array}$ & $\begin{array}{c}0.075 \\
(0.309)\end{array}$ & $\begin{array}{c}0.237 \\
(0.130)\end{array}$ & $\begin{array}{c}0.537 \\
(0.288)\end{array}$ \\
\hline$\alpha\left(\tau_{1}\right)$ & $\begin{array}{c}0.953 \\
(0.254) \\
\end{array}$ & $\begin{array}{c}0.938 \\
(0.191)\end{array}$ & $\begin{array}{c}0.934 \\
(0.108)\end{array}$ & $\begin{array}{c}0.920 \\
(0.227)\end{array}$ & $\begin{array}{l}0.453^{*} \\
(0.000)\end{array}$ & $\begin{array}{c}0.872 \\
(0.105)\end{array}$ & $\begin{array}{c}1.046 \\
(0.360)\end{array}$ & $\begin{array}{c}1.109 \\
(0.334)\end{array}$ & $\begin{array}{c}0.965 \\
(0.391)\end{array}$ & $\begin{array}{c}0.951 \\
(0.292)\end{array}$ & $\begin{array}{c}0.976 \\
(0.417)\end{array}$ & $\begin{array}{c}1.070 \\
(0.403) \\
\end{array}$ \\
\hline$\alpha\left(\tau_{n}\right)$ & \begin{tabular}{|l|}
-0.703 \\
$(0.480)$
\end{tabular} & $\begin{array}{l}-1.396 \\
(0.330)\end{array}$ & $\begin{array}{l}-1.048 \\
(0.340)\end{array}$ & $\begin{array}{l}-0.486 \\
(0.610)\end{array}$ & $\begin{array}{c}- \\
2.510^{* * *} \\
(0.060)\end{array}$ & $\begin{array}{l}-1.076 \\
(0.200)\end{array}$ & $\begin{array}{c}0.408 \\
(0.870)\end{array}$ & $\begin{array}{c}0.373 \\
(0.890)\end{array}$ & $\begin{array}{l}-0.216 \\
(0.610)\end{array}$ & $\begin{array}{l}-1.023 \\
(0.270)\end{array}$ & $\begin{array}{l}-0.405 \\
(0.490)\end{array}$ & $\begin{array}{c}0.138 \\
(0.890)\end{array}$ \\
\hline $\begin{array}{l}\text { QSK- } \\
\text { stat }\end{array}$ & \multicolumn{4}{|c|}{2.023 [4.619] } & \multicolumn{4}{|c|}{$\frac{1}{2.510[5.246]}$} & \multicolumn{4}{|c|}{$1.553[4.731]$} \\
\hline \multicolumn{13}{|c|}{ Panel 4: TOP } \\
\hline$\alpha\left(\tau_{0}\right)$ & \begin{tabular}{|l}
$-0.093^{*}$ \\
$(0.000)$
\end{tabular} & $\begin{array}{l}-0.028 \\
(0.120)\end{array}$ & $\begin{array}{c}0.026 \\
(0.263)\end{array}$ & $\begin{array}{l}0.225^{*} \\
(0.002)\end{array}$ & $\begin{array}{c}-0.159^{*} \\
(0.003)\end{array}$ & $\begin{array}{c}0.016 \\
(0.385)\end{array}$ & $\begin{array}{c}0.107^{* *} \\
(0.046)\end{array}$ & $\begin{array}{l}0.279 * \\
(0.000)\end{array}$ & $\begin{array}{c}- \\
0.049 * * * \\
(0.051)\end{array}$ & $\begin{array}{c}0.017 \\
(0.161)\end{array}$ & $\begin{array}{l}0.048^{*} \\
(0.001)\end{array}$ & \begin{tabular}{|l}
$0.090^{*}$ \\
$(0.000)$
\end{tabular} \\
\hline$\alpha\left(\tau_{1}\right)$ & $\begin{array}{c}0.908 \\
(0.132)\end{array}$ & $\begin{array}{c}0.867 * * * \\
(0.087)\end{array}$ & $\begin{array}{c}0.928 \\
(0.304)\end{array}$ & $\begin{array}{l}0.570^{*} \\
(0.050)\end{array}$ & $\begin{array}{c}0.854 \\
(0.134)\end{array}$ & $\begin{array}{c}0.845^{* * *} \\
(0.087)\end{array}$ & $\begin{array}{c}0.852 \\
(0.138)\end{array}$ & $\begin{array}{c}0.794 * * * \\
(0.059)\end{array}$ & $\begin{array}{c}0.884 \\
(0.272)\end{array}$ & $\begin{array}{c}0.922 \\
(0.246)\end{array}$ & $\begin{array}{c}0.921 \\
(0.212)\end{array}$ & $\begin{array}{c}0.964 \\
(0.406)\end{array}$ \\
\hline$\alpha\left(\tau_{n}\right)$ & $\begin{array}{l}-0.855 \\
(0.310) \\
\end{array}$ & $\begin{array}{l}-1.403 \\
(0.330)\end{array}$ & $\begin{array}{l}-0.411 \\
(0.630) \\
\end{array}$ & $\begin{array}{l}-1.513 \\
(0.260)\end{array}$ & $\begin{array}{l}-0.239 \\
(0.440)\end{array}$ & $\begin{array}{l}-1.377 \\
(0.290)\end{array}$ & $\begin{array}{l}-1.440 \\
(0.360) \\
\end{array}$ & $\begin{array}{l}-1.564 \\
(0.270)\end{array}$ & $\begin{array}{l}-0.651 \\
(0.600)\end{array}$ & $\begin{array}{l}-0.840 \\
(0.600) \\
\end{array}$ & $\begin{array}{l}-0.865 \\
(0.440) \\
\end{array}$ & $\begin{array}{l}-0.234 \\
(0.590) \\
\end{array}$ \\
\hline $\begin{array}{l}\text { QSK- } \\
\text { stat }\end{array}$ & \multicolumn{4}{|c|}{2.029 [5.529] } & \multicolumn{4}{|c|}{$2.584[3.829]$} & \multicolumn{4}{|c|}{$1.553[4.731]$} \\
\hline \multicolumn{13}{|c|}{ Panel 5: GEX } \\
\hline$\alpha\left(\tau_{0}\right)$ & \begin{tabular}{|c}
$-0.092^{*}$ \\
$(0.010)$
\end{tabular} & $\begin{array}{l}-0.026 \\
(0.110)\end{array}$ & $\begin{array}{c}0.028 \\
(0.129)\end{array}$ & $\begin{array}{c}0.054 \\
(0.108)\end{array}$ & $\begin{array}{c}-0.160^{*} \\
(0.002)\end{array}$ & $\begin{array}{l}-0.010 \\
(0.437)\end{array}$ & $\begin{array}{l}0.097 * \\
(0.002)\end{array}$ & $\begin{array}{l}0.394 * \\
(0.009)\end{array}$ & $\begin{array}{l}-0.027 \\
(0.198)\end{array}$ & $\begin{array}{c}0.024 * * \\
(0.042)\end{array}$ & $\begin{array}{l}0.032 * \\
(0.005)\end{array}$ & $\begin{array}{l}0.063^{*} \\
(0.001) \\
\end{array}$ \\
\hline$\alpha\left(\tau_{1}\right)$ & $\begin{array}{c}0.732^{* *} \\
(0.020) \\
\end{array}$ & $\begin{array}{c}0.926 \\
(0.147) \\
\end{array}$ & $\begin{array}{c}1.030 \\
(0.339) \\
\end{array}$ & $\begin{array}{c}1.025 \\
(0.428) \\
\end{array}$ & $\begin{array}{c}0.894 \\
(0.240) \\
\end{array}$ & $\begin{array}{c}1.023 \\
(0.424) \\
\end{array}$ & $\begin{array}{l}0.752^{*} \\
(0.000)\end{array}$ & $\begin{array}{l}0.007^{*} \\
(0.000)\end{array}$ & $\begin{array}{c}1.056 \\
(0.315) \\
\end{array}$ & $\begin{array}{c}0.892^{* *} \\
(0.036)\end{array}$ & $\begin{array}{c}0.907 * * * \\
(0.064) \\
\end{array}$ & \begin{tabular}{|c}
$0.752^{*}$ \\
$(0.007)$ \\
\end{tabular} \\
\hline$\alpha\left(\tau_{n}\right)$ & \begin{tabular}{|l}
$-5.759 *$ \\
\end{tabular} & -1.303 & 0.551 & 0.313 & -0.507 & 0.250 & - & $-4.648^{*}$ & 0.729 & -2.063 & $-2.436 * *$ & $-5.005^{*}$ \\
\hline
\end{tabular}




\begin{tabular}{|c|c|c|c|c|c|c|c|c|c|c|c|c|}
\hline & $(0.000)$ & $(0.280)$ & $(0.980)$ & $(0.900)$ & $(0.610)$ & $(0.960)$ & $\begin{array}{c}1.578^{* * *} \\
(0.060)\end{array}$ & $(0.010)$ & $(0.960)$ & $(0.100)$ & $(0.030)$ & $(0.000)$ \\
\hline $\begin{array}{l}\text { QSK- } \\
\text { stat }\end{array}$ & \multicolumn{4}{|c|}{8.532 [4.782] } & \multicolumn{4}{|c|}{$4.648[5.546]$} & \multicolumn{4}{|c|}{$5.341[4.646]$} \\
\hline \multicolumn{13}{|c|}{ Panel 6: PST } \\
\hline$\alpha\left(\tau_{0}\right)$ & $\begin{array}{c}0.016 \\
(0.452)\end{array}$ & $\begin{array}{l}0.058^{*} \\
(0.000)\end{array}$ & $\begin{array}{l}0.142^{*} \\
(0.000)\end{array}$ & $\begin{array}{l}0.208^{*} \\
(0.000)\end{array}$ & $\begin{array}{c}0.116 \\
(0.319)\end{array}$ & $\begin{array}{l}0.179 * \\
(0.002)\end{array}$ & $\begin{array}{l}0.294 * \\
(0.000)\end{array}$ & $\begin{array}{l}0.326^{*} \\
(0.000)\end{array}$ & $\begin{array}{c}0.009 \\
(0.450)\end{array}$ & $\begin{array}{c}0.062 \\
(0.238)\end{array}$ & $\begin{array}{l}0.085^{*} \\
(0.000)\end{array}$ & $\begin{array}{l}0.155^{*} \\
(0.000)\end{array}$ \\
\hline$\alpha\left(\tau_{1}\right)$ & $\begin{array}{c}1.006 \\
(0.470)\end{array}$ & $\begin{array}{l}0.962^{*} \\
(0.000)\end{array}$ & $\begin{array}{l}0.875^{*} \\
(0.000)\end{array}$ & $\begin{array}{l}0.826^{*} \\
(0.000)\end{array}$ & $\begin{array}{c}0.957 \\
(0.426)\end{array}$ & $\begin{array}{c}0.867^{* *} \\
(0.042)\end{array}$ & $\begin{array}{l}0.729 * \\
(0.000)\end{array}$ & $\begin{array}{l}0.751^{*} \\
(0.000)\end{array}$ & $\begin{array}{c}1.003 \\
(0.482)\end{array}$ & $\begin{array}{c}0.960 \\
(0.322)\end{array}$ & $\begin{array}{l}0.944^{*} \\
(0.000)\end{array}$ & $\begin{array}{l}0.892^{*} \\
(0.000)\end{array}$ \\
\hline$\alpha\left(\tau_{n}\right)$ & $\begin{array}{c}0.014 \\
(0.880)\end{array}$ & $\begin{array}{l}-3.172^{*} \\
(0.000)\end{array}$ & $\begin{array}{c}-7.212^{*} \\
(0.000)\end{array}$ & $\begin{array}{c}-13.319^{*} \\
(0.000)\end{array}$ & $\begin{array}{l}-0.120 \\
(0.320)\end{array}$ & $\begin{array}{c}-4.757^{*} \\
(0.000)\end{array}$ & $\begin{array}{c}-10.327^{*} \\
(0.000)\end{array}$ & $\begin{array}{l}-2.618^{*} \\
(0.000)\end{array}$ & $\begin{array}{c}0.520 \\
(0.970)\end{array}$ & $\begin{array}{c}-4.661^{*} \\
(0.000)\end{array}$ & $\begin{array}{l}-5.474^{*} \\
(0.000)\end{array}$ & $\begin{array}{l}-5.005^{*} \\
(0.000)\end{array}$ \\
\hline $\begin{array}{l}\text { QSK- } \\
\text { stat }\end{array}$ & \multicolumn{4}{|c|}{$13.319[3.445]$} & \multicolumn{4}{|c|}{$10.327[3.578]$} & \multicolumn{4}{|c|}{$5.474[3.136]$} \\
\hline
\end{tabular}

Note: *,** and *** indicate the significance level at 1, 5 and $10 \%$ respectively. QSK denotes the Kolmogorov-Smirvov test type proposed by Konker and Xiao (2004). The numbers in ( ) and [ ] represents the p-values and QSK critical values, respectively.

In the case of Egypt and Nigeria, the variable of COP, FD and TOP have a shock of good magnitude at the lower and upper quantile, while GEX shocked occurred at the lower quantile in Egypt, but at lower and upper quantile in Nigeria. For PST, the shocked occurs in the middle and upper quantiles in Egypt and Nigeria. However, for RGDP, the shocked occurs at lower quantile in Nigeria and South Africa. Interestingly, only South Africa does not have significant shock of any magnitude in the series FD. However, the results indicated that FD has the highest shock among the variables that cause the series to shift away from its long run equilibrium level of about 0.403 and 1.308 units in Egypt and Nigeria respectively. But in South African economy, it is the COP variable that has the highest shock that moves the series far away from the equilibrium level of about 0.188 units. The estimated values of $\alpha\left(\tau_{1}\right)$ indicated that the unit root null for Egypt and Nigeria for COP, TOP and PST are rejected at the middle and upper quantiles. For Nigeria and South Africa, the GEX series rejects the unit root null in higher quantiles. Interestingly, PST reject the unit root null in higher quantiles for all countries under study. These results indicate that for PST, there is stationary in higher quantiles that displays mean reversion.

Given the existence of the unit root test in both traditional and quantile estimates, we estimate the long run cointegration test. First, we used the Bayer and Hanck (2013) cointegration model of both EG$\mathrm{JOH}$ and EG-JOH-BO-BDM. The estimated results in Table 5 indicates clearly that, there is a rejection of the null hypothesis of no cointegration for EG-JOH combine cointegration test. However, by using the EG-JOH-BO estimation, we found the estimation model for Egypt rejects the null hypothesis of no cointegration at the $1 \%$ level of significance. Therefore, this implies that the combine cointegration test results accept the existence of the long run relationship between the variables.

Table 5

Bayer and Hanck (2013) combine cointegration test results

\begin{tabular}{|l|c|c|c|}
\hline \multicolumn{1}{|c|}{ Countries } & EG-JOH & EG-JOH-BO-BDM & Null hypothesis \\
\hline Egypt & $8.376^{* * *}$ & $26.606^{*}$ & Reject \\
\hline Nigeria & $9.651^{* * *}$ & 10.205 & Reject \\
\hline South Africa & $10.213^{* * *}$ & 11.484 & \\
\hline & \multicolumn{2}{|c|}{ Significance level } \\
\hline $10 \%$ & 8.242 & 15.804 & \\
\hline $5 \%$ & 10.419 & 19.888 & \\
\hline $1 \%$ & 15.701 & 29.85 & \\
\hline
\end{tabular}

Note: ${ }^{*}, * *$ and $* * *$ indicate the significance level at 1, 5 and $10 \%$ respectively. 
To strengthen our cointegration analysis, we now turn to the long run coefficient estimates using OLS, DOLS and FMOLS techniques as shown in Table 6. In the Egyptian economy, the OLS and FMOLS estimates captured negative relationship between FD and RGDP, while the DOLS estimates show a positive relationship between TOP and RGDP. Interestingly, these OLS, FMOLS and DOLS estimates proved a positive relationship between GEX and RGDP; and PST and RGDP. The estimation results for Nigeria shows that, the COP drives RGDP, while FD impedes it. The DOLS estimates indicate a negative relationship between TOP and RGDP, while positive relationship arises from GEX. In South Africa, the estimated results indicate a negative relationship of COP and GEX., It implies that COP and GEX stymied growth in South Africa. While TOP is positively related to RGDP according to DOLS estimates; and suggest a positive relationship of PST.

Table 6

FMOLS and DOLS cointegration test results

\begin{tabular}{|l|c|c|c|c|c|c|c|c|c|}
\hline & \multicolumn{3}{|c|}{ OLS } & \multicolumn{3}{c|}{ FMOLS } & \multicolumn{3}{c|}{ DOLS } \\
\cline { 2 - 10 } & Egypt & Nigeria & $\begin{array}{l}\text { South } \\
\text { Africa }\end{array}$ & Egypt & Nigeria & $\begin{array}{l}\text { South } \\
\text { Africa }\end{array}$ & Egypt & Nigeria & $\begin{array}{c}\text { South } \\
\text { Africa }\end{array}$ \\
\hline COP & 0.205 & $1.136^{*}$ & $-0.597^{*}$ & 0.243 & $1.580^{* * *}$ & $-0.739^{*}$ & -0.113 & 1.561 & $-1.515^{*}$ \\
& $(0.155)$ & $(0.044)$ & $(0.000)$ & $(0.115)$ & $(0.063)$ & $(0.001)$ & $(0.672)$ & $(0.350)$ & $(0.001)$ \\
\hline FD & $-0.076^{*}$ & $-0.390^{*}$ & $-0.083^{*}$ & $-0.066^{*}$ & $-0.427^{* *}$ & -0.055 & -0.035 & -0.430 & 0.099 \\
& $(0.007)$ & $(0.004)$ & $(0.016)$ & $(0.027)$ & $(0.038)$ & $(0.226)$ & $(0.243)$ & $(0.193)$ & $(0.324)$ \\
\hline TOP & 0.47 & $-1.532^{*}$ & 0.019 & 0.211 & $-2.203^{* *}$ & 0.419 & $0.531^{* * *}$ & $-2.873^{* *}$ & $1.498^{* * *}$ \\
& $(0.413)$ & $(0.013)$ & $(0.961)$ & $(0.279)$ & $(0.022)$ & $(0.432)$ & $(0.095)$ & $(0.047)$ & $(0.071)$ \\
\hline GEX & $1.734^{*}$ & $3.957^{*}$ & $-2.599^{*}$ & $1.913^{*}$ & $4.863^{*}$ & $-2.545^{*}$ & $1.754^{*}$ & $4.683^{* *}$ & $-2.105^{*}$ \\
& $(0.000)$ & $(0.000)$ & $(0.000)$ & $(0.000)$ & $(0.000)$ & $(0.000)$ & $(0.000)$ & $(0.048)$ & $(0.000)$ \\
\hline PST & $0.106^{*}$ & -0.199 & $0.164^{*}$ & $0.113^{*}$ & -0.175 & $0.174^{*}$ & $0.157^{*}$ & -0.484 & $0.259^{*}$ \\
& $(0.001)$ & $(0.453)$ & $(0.000)$ & $(0.001)$ & $(0.662)$ & $(0.000)$ & $(0.002)$ & $(0.549)$ & $(0.002)$ \\
\hline
\end{tabular}

Note: $* * *$ and $* * *$ indicate significance at 1,5 and $10 \%$ respectively. Value in () represent the p-values

The final part the estimation results of this study will be the quantile bootstrap regression (see Table 7). Typically, in Egypt the main driver of economic growth is GEX, which is significant in all quantiles with coefficient ranging between 2.178 to 2.066. The participation of government in the economy remains the major driver of the economy, while the private sector is poor considering the impact of COP and FD. On the PST, it has a positive relationship to economic growth in the middle and higher quantiles. It implies that the unstable political institution as a result of Arab Spring has no negative impact on growth. This is contrary to the finding of Gong and Rao (2016), that found a negative impact on economic growth in Fiji economy, as a result of the unstable political situation. Also, Okafor (2017) found poor governance in the form of political instability undermining economic growth among the ECOWAS countries. In fact, Arayssi and Fakih (2017) also contributed that, in the pre-Arab Spring period, macroeconomic variables were enhancing economic growth, but in the post-Arab Spring period they undermine economic growth because of the unstable political situation in the MENA countries. Meanwhile, the square series of FD suggests a negative relationship with economic growth, indicating lag in the desired threshold for FD to impact on growth for Egypt. This result is in line with Roll (2010) that argued the Egypt financial reform policy has not impacted the generality of the Egyptian populace in business, and only a few entrepreneurial elites who are connected to the political class, such people gain credit easily from the financial sector.

The Nigerian economy proof palpable to grow as a result of capital flows, as the relationship between COP and economic growth is positive at the lower and upper quantile within the range of 1.325 to 1.819 . 
This demonstrates Nigerian economy has the capacity to benefit more on capital flows as the coefficient is increasing. We found, the financial liberalization policies of 1986 and the Foreign Exchange Act in 1995 (Monitoring and Miscellaneous Provision), emphasized the removal of restriction on foreign exchange transactions is working well for Nigeria. In the case of FD, the coefficient shows a negative and statistically significant in the lower and upper quantiles. Similarly, the squared coefficient of FD is not significant, which is contrary to the results of Adeniyi et al, (2015) from a study involve Nigeria. We found; the TOP is negatively related to economic growth at lower quantile. Young (1991) study found the finance-growth nexus, and the trade openness is undermining growth in the situation where international trade affects infant industries. This was the case in Nigeria as the import in the 1970s and the 80s went astronomically high and was not supporting the local industries of the country. The PST is negatively related to economic growth, this may be an evidence why FD cannot impact on economic growth, and more reflecting on COP can as find by Klein and Olivei (2008).

The estimated results for South Africa suggested that, the FD has impeded economic growth contrary to the findings of Polat, et al (2014) and Salahuddin and Grow (2016). However, when the FD variable is squared the relationship become positive and significant. This is an evidence that at the threshold FD impact on growth. This agrees with the results found by Ibrahim and Alagidede (2017), suggesting financial development advanced will more impactful towards the economic growth. While, Hook and Singh (2014); and Cecchetti and Kharroubi (2012) found an inverted U-shaped relationship between the finance and growth relationship. Typically, TOP at upper quantile shows a negative relationship with economic growth. This agreed with the results of Gossel and Biekpe (2014) that found export trade, stimulating growth, while import trade stymied growth in South Africa. At the upper quantile the PST enhances economic growth, which can be connected to the favorable economic situation the South African economy experience before the corruption scandal of President Jacob Zuma, which finally led to his resignation in 2018.

Table 7

Bootstrap quantile regression results

\begin{tabular}{|c|c|c|c|c|c|c|c|c|c|c|c|c|}
\hline & \multicolumn{4}{|c|}{ Egypt } & \multicolumn{4}{|c|}{ Nigeria } & \multicolumn{4}{|c|}{ South Africa } \\
\hline & q25 & $q 50$ & $\mathrm{q} 75$ & q90 & q25 & q50 & q75 & q90 & q25 & q50 & q75 & q90 \\
\hline COP & $\begin{array}{c}0.192 \\
(0.659)\end{array}$ & $\begin{array}{l}-0.096 \\
(0.530)\end{array}$ & $\begin{array}{l}-0.048 \\
(0.804)\end{array}$ & $\begin{array}{c}0.171 \\
(0.614)\end{array}$ & $\begin{array}{c}1.819^{* * *} \\
(0.071)\end{array}$ & $\begin{array}{c}1.325 \\
(0.173)\end{array}$ & $\begin{array}{c}1.335^{* * *} \\
(0.057)\end{array}$ & $\begin{array}{c}0.074 \\
(0.939)\end{array}$ & $\begin{array}{l}-0.163 \\
(0.366)\end{array}$ & $\begin{array}{l}-0.126 \\
(0.560)\end{array}$ & $\begin{array}{c}0.085 \\
(0.700)\end{array}$ & $\begin{array}{l}-0.366 \\
(0.256)\end{array}$ \\
\hline FD & $\begin{array}{l}-0.044 \\
(0.295)\end{array}$ & $\begin{array}{l}-0.028 \\
(0.388)\end{array}$ & $\begin{array}{l}-0.034 \\
(0.455)\end{array}$ & $\begin{array}{l}-0.066 \\
(0.432)\end{array}$ & $\begin{array}{c}-0.595^{* *} \\
(0.055)\end{array}$ & $\begin{array}{l}-0.369 \\
(0.133)\end{array}$ & $\begin{array}{c}-0.417 * * \\
(0.044)\end{array}$ & $\begin{array}{l}-0.266 \\
(0.215)\end{array}$ & $\begin{array}{c}-0.330^{*} \\
(0.000)\end{array}$ & $\begin{array}{c}-0.299 * \\
(0.001)\end{array}$ & $\begin{array}{l}-0.290^{*} \\
(0.001)\end{array}$ & $\begin{array}{c}-0.181^{* * *} \\
(0.085)\end{array}$ \\
\hline $\mathrm{FD}^{2}$ & $\begin{array}{l}-0.049 * \\
(0.030)\end{array}$ & $\begin{array}{l}-0.049^{*} \\
(0.005)\end{array}$ & $\begin{array}{c}-0.048^{* *} \\
(0.019)\end{array}$ & $\begin{array}{l}-0.038 \\
(0.105)\end{array}$ & $\begin{array}{c}0.069 \\
(0.378)\end{array}$ & $\begin{array}{l}-0.016 \\
(0.811)\end{array}$ & $\begin{array}{l}-0.054 \\
(0.334)\end{array}$ & $\begin{array}{l}-0.078 \\
(0.206)\end{array}$ & $\begin{array}{l}0.099 * \\
(0.001)\end{array}$ & $\begin{array}{c}0.081^{\text {** }} \\
(0.014)\end{array}$ & $\begin{array}{c}0.072^{* *} \\
(0.034)\end{array}$ & $\begin{array}{c}0.020 \\
(0.654)\end{array}$ \\
\hline TOP & $\begin{array}{l}-0.048 \\
(0.936)\end{array}$ & $\begin{array}{c}0.326 \\
(0.174)\end{array}$ & $\begin{array}{c}0.203 \\
(0.293)\end{array}$ & $\begin{array}{l}-0.063 \\
(0.779)\end{array}$ & $\begin{array}{c}-2.264^{*} \\
(0.001)\end{array}$ & $\begin{array}{l}-1.084 \\
(0.257)\end{array}$ & $\begin{array}{c}0.416 \\
(0.658)\end{array}$ & $\begin{array}{c}0.415 \\
(0.734)\end{array}$ & $\begin{array}{l}-0.402 \\
(0.261)\end{array}$ & $\begin{array}{l}-0.361 \\
(0.379)\end{array}$ & $\begin{array}{c}-0.822^{* * *} \\
(0.094)\end{array}$ & $\begin{array}{l}-0.083 \\
(0.871)\end{array}$ \\
\hline$\overline{\text { GEX }}$ & $\begin{array}{l}2.178^{*} \\
(0.000)\end{array}$ & $\begin{array}{l}2.066^{*} \\
(0.000)\end{array}$ & $\begin{array}{l}2.136^{*} \\
(0.000)\end{array}$ & $\begin{array}{l}1.899 * \\
(0.000)\end{array}$ & $\begin{array}{c}3.516^{* *} \\
(0.021)\end{array}$ & $\begin{array}{l}4.835^{*} \\
(0.000)\end{array}$ & $\begin{array}{l}5.092^{*} \\
(0.000)\end{array}$ & $\begin{array}{c}3.046^{* * *} \\
(0.070)\end{array}$ & $\begin{array}{l}-2.384^{*} \\
(0.000)\end{array}$ & $\begin{array}{l}-2.212^{*} \\
(0.000)\end{array}$ & $\begin{array}{l}-2.632^{*} \\
(0.000)\end{array}$ & $\begin{array}{l}-2.183^{*} \\
(0.000)\end{array}$ \\
\hline$\overline{\text { PST }}$ & $\begin{array}{c}0.071 \\
(0.211)\end{array}$ & $\begin{array}{l}0.114^{*} \\
(0.000)\end{array}$ & $\begin{array}{l}0.145^{*} \\
(0.000)\end{array}$ & $\begin{array}{l}0.139^{*} \\
(0.001)\end{array}$ & $\begin{array}{c}0.214 \\
(0.568)\end{array}$ & $\begin{array}{l}-0.021 \\
(0.964)\end{array}$ & $\begin{array}{l}-0.086 \\
(0.865)\end{array}$ & $\begin{array}{l}-0.279 \\
(0.678)\end{array}$ & $\begin{array}{c}0.020 \\
(0.629)\end{array}$ & $\begin{array}{c}0.055 \\
(0.315)\end{array}$ & $\begin{array}{c}0.070 \\
(0.186)\end{array}$ & $\begin{array}{c}0.136^{* *} \\
(0.033)\end{array}$ \\
\hline
\end{tabular}

Note: *,** and $* * *$ indicate significance at 1, 5 and $10 \%$ respectively. Figures in () are the p-values

The following figures give the trend coefficients of the different quantile in this study, which depicts the influence of the independent variables on the RGDP in respect to their quantiles. For Egypt, the COP indicates an unstable moment in the quantiles 0.30 and 0.80 . The FD trended downward with unstable moment at 0.8 quantile, while the $95 \%$ confidence interval of TOP trended upward with unstable 
condition at 0.30 quantile. On the coefficient of the GEX, there was an unstable condition at 0.30 and 0.80 quantile, while PST is at quantile 0.30 and the square of FD trended under stable condition on all quantile, at 0.80 quantile. In case of Nigeria, the COP series shows unstable state only at quantile 0.40 and 0.50 and FD trended upward with unstable condition at quantile 0.2. The TOP has mixed condition at 0.80 to 0.90 quantile. The coefficient of GEX is unstable at 0.40 quantile, while PST is unstable at quantile 0.90 , but the squared of FD is relatively stable. For South Africa, the COP series has an unstable condition at 0.80 quantile, while FD, PST and FD squared series are relatively stable. For TOP and GEX there is an unstable condition at 0.80 quantile.

Next, we examine the complementary or otherwise of the interactive variables. The estimated results for Egypt showed that the interaction of FD and COP impede economic growth at middle and higher quantile (see Table 8). This agrees with Roll (2010) who contributed that the financial reform program is marred with malpractice and government still control the financial market to a few people advantage. We found, Nigeria had a high inflow of capital, but that has no influence on the advancement of domestic financial market, as the interaction effects of FD with COP stymied economic growth at the upper quantile. This supports the findings that the domestic financial system in Nigeria is still not at the advanced stage to stimulate economic growth as found by Adeniyi et al. (2015). Interestingly, for South Africa, there is a complementary relationship between FD and COP to economic growth. This support the squared coefficient of FD of having a positive relationship with economic growth, implying that in the different quantile's capital inflows advanced domestic financial markets. Similar results were found by Ahmed (2016) in Sub Sahara Africa countries. However, the interaction effects of FD with TOP are not palpable to economic growth at the upper quantile in South Africa, contrary to the contribution of Pan et al. (2019) that in a liberalized financial system, financial development and trade openness spur economic growth.

Table 8

Bootstrap quantile regression with interaction effects

\begin{tabular}{|c|c|c|c|c|c|c|c|c|c|c|c|c|}
\hline & \multicolumn{4}{|c|}{ Egypt } & \multicolumn{4}{|c|}{ Nigeria } & \multicolumn{4}{|c|}{ South Africa } \\
\hline & q25 & $q 50$ & q75 & q90 & q25 & $q 50$ & q75 & $q 90$ & $\mathrm{q} 25$ & $q 50$ & q75 & q90 \\
\hline COP & $\begin{array}{l}-0.169 \\
(0.431)\end{array}$ & $\begin{array}{l}-0.127 \\
(0.463)\end{array}$ & $\begin{array}{l}-0.081 \\
(0.564)\end{array}$ & $\begin{array}{c}-0.132 \\
(0.305)\end{array}$ & $\begin{array}{l}2.375^{*} \\
(0.006)\end{array}$ & $\begin{array}{l}2.752^{*} \\
(0.009)\end{array}$ & $\begin{array}{c}1.890^{* * *} \\
(0.064)\end{array}$ & $\begin{array}{l}2.646^{* *} \\
(0.011)\end{array}$ & $\begin{array}{c}-0.897^{*} \\
(0.000)\end{array}$ & $\begin{array}{c}-0.815^{*} \\
(0.001)\end{array}$ & $\begin{array}{c}-0.999 * \\
(0.001)\end{array}$ & $\begin{array}{l}-0.776^{*} \\
(0.007)\end{array}$ \\
\hline FD & $\begin{array}{l}-1.149 \\
(0.230) \\
\end{array}$ & $\begin{array}{l}-0.186 \\
(0.724) \\
\end{array}$ & $\begin{array}{l}-0.303 \\
(0.493) \\
\end{array}$ & $\begin{array}{l}-0.166 \\
(0.715) \\
\end{array}$ & $\begin{array}{l}-1.763 \\
(0.119) \\
\end{array}$ & $\begin{array}{l}-2.028 \\
(0.133) \\
\end{array}$ & $\begin{array}{l}-0.389 \\
(0.819) \\
\end{array}$ & $\begin{array}{l}-1.635 \\
(0.297) \\
\end{array}$ & $\begin{array}{c}1.238 \\
(0.320) \\
\end{array}$ & $\begin{array}{c}0.439 \\
(0.560) \\
\end{array}$ & $\begin{array}{c}1.600 \\
(0.231) \\
\end{array}$ & $\begin{array}{c}1.377 \\
(0.121) \\
\end{array}$ \\
\hline TOP & $\begin{array}{c}0.360 \\
(0.293)\end{array}$ & $\begin{array}{c}0.286 \\
(0.269)\end{array}$ & $\begin{array}{c}0.170 \\
(0.504)\end{array}$ & $\begin{array}{c}-0.012 \\
(0.953)\end{array}$ & $\begin{array}{c}-2.644^{*} \\
(0.003)\end{array}$ & $\begin{array}{c}-2.391^{* *} \\
(0.013)\end{array}$ & $\begin{array}{l}-0.338 \\
(0.751)\end{array}$ & $\begin{array}{l}-0.417 \\
(0.650)\end{array}$ & $\begin{array}{l}-0.620 \\
(0.364)\end{array}$ & $\begin{array}{c}-0.250 \\
(0.614)\end{array}$ & $\begin{array}{l}-0.017 \\
(0.977)\end{array}$ & $\begin{array}{l}-0.113 \\
(0.798)\end{array}$ \\
\hline GEX & $\begin{array}{l}1.948^{*} \\
(0.002)\end{array}$ & $\begin{array}{l}2.001^{*} \\
(0.000)\end{array}$ & $\begin{array}{l}2.115^{*} \\
(0.000)\end{array}$ & $\begin{array}{l}2.445^{*} \\
(0.000)\end{array}$ & $\begin{array}{l}4.823^{*} \\
(0.000)\end{array}$ & $\begin{array}{l}5.752^{*} \\
(0.000)\end{array}$ & $\begin{array}{l}6.000^{*} \\
(0.000)\end{array}$ & $\begin{array}{l}6.361^{*} \\
(0.000)\end{array}$ & $\begin{array}{c}-3.059^{*} \\
(0.000)\end{array}$ & $\begin{array}{c}-2.109^{*} \\
(0.000)\end{array}$ & $\begin{array}{c}-2.034^{*} \\
(0.000)\end{array}$ & $\begin{array}{l}-2.000^{*} \\
(0.000)\end{array}$ \\
\hline PST & $\begin{array}{l}0.125^{*} \\
(0.007)\end{array}$ & $\begin{array}{l}0.145^{*} \\
(0.000)\end{array}$ & $\begin{array}{l}0.127^{*} \\
(0.002)\end{array}$ & $\begin{array}{c}0.113^{*} \\
(0.003)\end{array}$ & $\begin{array}{c}0.322 \\
(0.375)\end{array}$ & $\begin{array}{c}0.095 \\
(0.858)\end{array}$ & $\begin{array}{l}-0.200 \\
(0.644)\end{array}$ & $\begin{array}{l}-0.149 \\
(0.683)\end{array}$ & $\begin{array}{c}0.012^{* *} \\
(0.044)\end{array}$ & $\begin{array}{c}0.209^{*} \\
(0.000)\end{array}$ & $\begin{array}{l}0.177^{*} \\
(0.001)\end{array}$ & $\begin{array}{l}0.161^{*} \\
(0.002)\end{array}$ \\
\hline $\begin{array}{l}\text { FD } \\
\text { COP) }\end{array}$ & $\begin{array}{l}-0.296 \\
(0.226)\end{array}$ & $\begin{array}{c}-0.144 * * * \\
(0.098)\end{array}$ & $\begin{array}{c}-0.188^{*} \\
(0.017)\end{array}$ & $\begin{array}{c}-0.275^{*} \\
(0.002)\end{array}$ & $\begin{array}{l}-0.460 \\
(0.420)\end{array}$ & $\begin{array}{l}-0.770 \\
(0.178)\end{array}$ & $\begin{array}{l}-0.819 \\
(0.125)\end{array}$ & $\begin{array}{c}-0.943 * * * \\
(0.077)\end{array}$ & $\begin{array}{c}0.304^{* *} \\
(0.034)\end{array}$ & $\begin{array}{c}0.195 \\
(0.106)\end{array}$ & $\begin{array}{c}0.359 * * \\
(0.043)\end{array}$ & $\begin{array}{c}0.263^{* * *} \\
(0.066)\end{array}$ \\
\hline $\begin{array}{l}\text { (FD } \\
\text { TOP) }\end{array}$ & $\begin{array}{c}0.275 \\
(0.260)\end{array}$ & $\begin{array}{c}0.033 \\
(0.801)\end{array}$ & $\begin{array}{c}0.059 \\
(0.591)\end{array}$ & $\begin{array}{c}0.026 \\
(0.817)\end{array}$ & $\begin{array}{c}0.303 \\
(0.299)\end{array}$ & $\begin{array}{c}0.295 \\
(0.376)\end{array}$ & $\begin{array}{l}-0.173 \\
(0.663)\end{array}$ & $\begin{array}{c}0.116 \\
(0.743)\end{array}$ & $\begin{array}{l}-0.305 \\
(0.330)\end{array}$ & $\begin{array}{c}-0.124 \\
(0.515)\end{array}$ & $\begin{array}{l}-0.413 \\
(0.217)\end{array}$ & $\begin{array}{c}-0.368^{* * *} \\
(0.099)\end{array}$ \\
\hline
\end{tabular}

Note: *, ** and ${ }^{* * *}$ indicate the significance level at 1, 5 and $10 \%$ respectively. Figure in () are p-values.

\section{CONCLUDING REMARKS}

Overall, we found the Egypt economy is still a public sector led economy, as government expenditure remains the major driver of economic growth. Thus, the financial reform policy of the 1990s is not impacting on economic growth through capital account openness and financial development. Policymakers should emphasize stable fiscal and monetary on the reduction of government involvement 
in the domestic financial market. Nigeria case is peculiar in terms of the impact of capital account openness to growth. Therefore, the 1995 Foreign Exchange Monitoring and Miscellaneous Act that liberalized capital account transaction is a step in the right direction. The political environment should only be stable to influence economic growth as that can equally improve capital inflows. The South African economy has a nonlinear relationship between financial development and economic growth. This means that, the expected threshold at which financial development is expected to influence economic growth is attended. The interaction of financial development with capital account openness is growth enhancing at all estimated quantiles. However, the government expenditure impedes economic growth in South Africa. This is because the Broad Based Black Economic Empowerment in the 2006 Accelerated and Shared Growth Initiative for South Africa (AsgiSA) aimed at promoting the emergence of the black capitalist. Therefore, the policy makers should take note and formulate policy that will not undermine growth.

Worthy of note also, is the AfCFTA that is aimed at increasing intra trade in Africa. The cutting of tariffs by $90 \%$ and harmonizing trading rules at a regional and continental level of goods and services if successful, AfCFTA is expected to boost intra-African trade by $50 \%$ by 2022 . This is very crucial for Africa and the countries under study especially. For centuries Europe, the United States, and recently China have stripped the continent of its raw materials. Since over $75 \%$ of Africa's external exports are raw materials such as oil and minerals. Interestingly, shifting away from the volatility associated with raw material exports towards industrial goods is hoping to secure sustainable economic growth in Africa. Similarly, amid growing United State-China tensions and China's concerns to reduce its reliance on export markets, is a sign that Africa is a prime successor to become the manufacturing hub of the developing world. These countries under study are among the leading economies in Africa as such should strive to take the opportunity to spur their economies.

\section{REFERENCES}

Abiad, A., Oomes, N., \& Ueda, K. (2008). The quality effect: Does financial liberalization improve the allocation of capital?. Journal of Development Economics, 87(2), 270-282.

Adeniyi, O., Oyinlola, A., Omisakin, O., \& Egwaikhide, F. O. (2015). Financial development and economic growth in Nigeria: Evidence from threshold modelling. Economic Analysis and Policy, 47, 11-21.

Ahmed, A. D. (2016). Integration of financial markets, financial development and growth: Is Africa different?. Journal of International Financial Markets, Institutions and Money, 42, 43-59.

Assefa, T. A., \& Mollick, A. V. (2017). Financial development and economic growth in Africa. Journal of African Business, 18(3), 320-339.

Arayssi, M., \& Fakih, A. (2017). Finance-growth nexus in a changing political region: How important was the Arab Spring?. Economic Analysis and Policy, 55, 106-123.

Bussiere, M., \& Fratzscher, M. (2008). Financial openness and growth: Short run gain, long-run pain. Review of International Economics, 16(1), 69-95.

Banerjee, A., Dolado, J., \& Mestre, R. (1998). Error-correction mechanism tests for cointegration in a single-equation framework. Journal of time series analysis, 19(3), 267-283.

Bayer, C., \& Hanck, C. (2013). Combining non-cointegration tests. Journal of Time Series Analysis, 34(1), 83-95.

Bilan, Y., Mishchuk, H., Samoliuk, N., \& Grishnova, O. (2019a). ICT and Economic Growth: Links and Possibilities of Engaging. Intellectual Economics, 13(1), 93-104.

Bilan, Y., Streimikiene, D., Vasylieva, T., Lyulyov, O., Pimonenko, T., \& Pavlyk, A. (2019b). Linking between Renewable Energy, CO2 Emissions, and Economic Growth: Challenges for Candidates and Potential Candidates for the EU Membership. Sustainability, 11(6), 1528.

Boswijk, H. P. (1994). Testing for an unstable root in conditional and structural error correction models. Journal of econometrics, 63(1), 37-60. 
Cecchetti, S. G., \& Kharroubi, E. (2012). Reassessing the impact of finance on growth. Reserve Bank of India's Second International Research Conference in Mumbai, India, on 1-2 February 2012.

Danlami, M. R., Loganathan, N. Streimikiene, D., Mardani, A., \& Golam Hassan, A.A. (2018). The effects of financial development and trade openness on Nigeria's dynamic growth. Economics and Sociology, 11(4), 128141. doi:10.14254/2071-789X.2018/11-4/8

De Haan, J., \& Sturm, J. E. (2017). Finance and income inequality: A review and new evidence. European Journal of Political Economy, 50, 171-195.

Demirgüç-Kunt, A., \& Maksimovic, V. (1998). Law, finance, and firm growth. The Journal of Finance, $53(6), 2107-$ 2137.

Dickey, D. A., \& Fuller, W. A. (1981). Likelihood ratio statistics for autoregressive time series with a unit root. Econometrica: Journal of the Econometric Society, 1057-1072.

Engle, R. F., \& Granger, C. W. (1987). Co-integration and error correction: representation, estimation, and testing. Econometrica: journal of the Econometric Society, 251-276.

Elkhuizen, L., Hermes, N., Jacobs, J., \& Meesters, A. (2018). Financial development, financial liberalization and social capital. Applied Economics, 50(11), 1268-1288.

Fotoyi, A. Tetani, S., \& Tsedu, M. (2016). Analysis of existing industrial policies and the state of implementation in South Africa. Trade and Industrial Policy Strategies. Available at: www.tips.org.za/

Fowowe, B. (2013). Financial liberalization in Sub-Saharan Africa: What do we know?. Journal of Economic Surveys, 27(1), 1-37.

Ghosh, S. (2019). Environmental Pollution, Income Inequality, and Household Energy Consumption: Evidence from the United Kingdom. Journal of International Commerce, Economics and Policy, 10(02), 1950008.

Gong, X., \& Rao, M. (2016). The economic impact of prolonged political instability: a case study of Fiji. Policy Studies, 37(4), 370-386.

Gossel, S. J., \& Biekpe, N. (2014). Economic growth, trade and capital flows: A causal analysis of post-liberalised South Africa. The Journal of International Trade \& Economic Development, 23(6), 815-836.

Newbold, P., \& Granger, C. W. J. (1974). Spurious regressions in econometrics. Journal of Econometrics, 2(2), 111-120.

Hermes, N., \& Lensink, R. (2003). Foreign direct investment, financial development and economic growth. The Journal of Development Studies, 40(1), 142-163.

Herwartz, H., \& Walle, Y. M. (2014). Openness and the finance-growth nexus. Journal of Banking \& Finance, 48, 235247.

Law, S. H., \& Singh, N. (2014). Does too much finance harm economic growth?. Journal of Banking \& Finance, 41, 3644.

Hu, H., Xie, N., Fang, D., \& Zhang, X. (2018). The role of renewable energy consumption and commercial services trade in carbon dioxide reduction: Evidence from 25 developing countries. Applied energy, 211, 1229-1244.

Ibrahim, M., \& Alagidede, P. (2018). Nonlinearities in financial development-economic growth nexus: Evidence from sub-Saharan Africa. Research in International Business and Finance, 46, 95-104.

Ibrahim, M., \& Alagidede, P. (2018). Effect of financial development on economic growth in sub-Saharan Africa. Journal of Policy Modeling, 40(6), 1104-1125.

Johansen, S. (1988). Statistical analysis of cointegration vectors. Journal of economic dynamics and control, 12(2-3), 231-254.

Koenker, R., \& Xiao, Z. (2004). Unit root quantile autoregression inference. Journal of the American Statistical Association, 99(467), 775-787.

King, R. G., \& Levine, R. (1993). Finance and growth: Schumpeter might be right. The quarterly journal of economics, 108(3), 717-737.

Klein, M. W., \& Olivei, G. P. (2008). Capital account liberalization, financial depth, and economic growth. Journal of international money and finance, 27(6), 861-875.

Korauš, A., Simionescu, M., Bilan, Y., \& Schönfeld, J. (2017). The Impact of Monetary Variables on the Economic Growth and Sustainable Development: Case of Selected Countries. Journal of Security \& Sustainability Issues, 6(3), 384-390.

Kunieda, T., Okada, K., \& Shibata, A. (2014). Corruption, capital account liberalization, and economic growth: Theory and evidence. International Economics, 139, 80-108. 
Lane, P., \& Milesi-Ferretti, G.M. (2014). Global imbalances and external adjustment after the crisis. IMF Working Papers. available at: https://doi.org/10.5089/9781498339216.001 (accessed on 10 December, 2018).

Law, S. H., Tan, H. B., \& Azman-Saini, W. N. W. (2014). Financial development and income inequality at different levels of institutional quality. Emerging Markets Finance and Trade, 50(sup1), 21-33.

Lin, B., \& Benjamin, N. I. (2017). Influencing factors on carbon emissions in China transport industry. A new evidence from quantile regression analysis. Journal of cleaner production, 150, 175-187.

Marshall, M. G., Gurr, T. R., \& Jaggers, K. (2016). Polity IV project dataset users' manual, v. 2015. Polity IV Project, 1 86. Retrieved from https://doi.org/10.1177/073889421349963

Mubi, S. (2012) 'Capital flows, trade openness and economic growth dynamics: New empirical evidence from Nigerian economy', University of Johannesburg, South Africa.

Nawaz, K., Lahiani, A., \& Roubaud, D. (2019). Natural resources as blessings and finance-growth nexus: A bootstrap ARDL approach in an emerging economy. Resources Policy, 60, 277-287.

Ngugi, R. W., \& Kabubo, J. W. (1998). Financial sector reforms and interest rate liberalization: The Kenya experience. AERC, Nairobi, KE.

Njikam, O. (2017). Financial liberalization and growth in African economies: The role of policy complementarities. Review of development finance, 7(1), 73-83.

Pan, X., Uddin, M. K., Han, C., \& Pan, X. (2019). Dynamics of financial development, trade openness, technological innovation and energy intensity: Evidence from Bangladesh. Energy, 171, 456-464.

Phillips, P. C., \& Hansen, B. E. (1990). Statistical inference in instrumental variables regression with I (1) processes. The Review of Economic Studies, 57(1), 99-125.

Phillips, P.C.B., \& Perron, P. (1988). Testing for unit roots in time series regression. Biometrika, 75(2), 335-346.

Polat, A., Shahbaz, M., Rehman, I. U., \& Satti, S. L. (2015). Revisiting linkages between financial development, trade openness and economic growth in South Africa: fresh evidence from combined cointegration test. Quality \& Quantity, 49(2), 785-803.

Prasad, E., Rogoff, K., Wei, S. J., \& Kose, M. A. (2005). Effects of financial globalization on developing countries: some empirical evidence. In India's and China's recent experience with reform and growth (pp. 201-228). Palgrave Macmillan, London.

Okafor, G. (2017). The impact of political instability on the economic growth of ECOWAS member countries. Defence and Peace Economics, 28(2), 208-229.

Onanuga, A. T., \& Onanuga, O. T. (2016). Do Financial and Trade Openness Lead to Financial Sector Development in Nigeria?. Zagreb International Review of Economics and Business, 19(2), 57-68.

Onyeiwu, C., \& Oladimeji, O.P. (2018). Impact of oil price on Nigerian economy. International Journal of Economics, Commerce and Management, 6(4), 252-264.

Rajan, R.G., \& Zingales, L. (1998). Financial dependence and growth. America Economic Review, 88(3), 559-586.

Rajan, R. G., \& Zingales, L. (2003). The great reversals: the politics of financial development in the twentieth century. Journal of financial economics, 69(1), 5-50.

Roll, S. (2010). 'Finance matters!'The influence of financial sector reforms on the development of the entrepreneurial elite in Egypt. Mediterranean Politics, 15(3), 349-370.

Salahuddin, M., \& Gow, J. (2016). The effects of Internet usage, financial development and trade openness on economic growth in South Africa: A time series analysis. Telematics and Informatics, 33(4), 1141-1154.

Shaw, E.S. (1973). Financial Deepening in Economic Development. New York: Oxford University Press.

Simionescu, M., Bilan, Y., \& Štreimikiené, D. (2019). The impact of biodiesel consumption by transport on economic growth in the European Union. Inæ̌inerine ekonomika, 50-58.

Stock, J. H., \& Watson, M. W. (1993). A simple estimator of cointegrating vectors in higher order integrated systems. Econometrica: Journal of the Econometric Society, 783-820.

Uddin, G. S., Sjö, B., \& Shahbaz, M. (2013). The causal nexus between financial development and economic growth in Kenya. Economic Modelling, 35, 701-707.

Xu, X., \& Yan, Y. (2014). Does government investment crowd out private investment in China?. Journal of Economic Policy Reform, 17(1), 1-12.

World Bank. (2019). World Development Indicator. Retrieved from http:www.worldbank.org/ 
Young, A. (1991). Learning by doing and the dynamic effects of international trade. The Quarterly Journal of Economics, 106(2), 369-405.

Zivot, E., \& Andrews, D. W. K. (2002). Further evidence on the great crash, the oil-price shock, and the unit-root hypothesis. Journal of business \& economic statistics, 20(1), 25-44. 